\title{
Process of community engagement in a mixed model research: A success story from Kolkata India
}

\author{
Arunansu Talukdar ${ }^{1}$, Ananya Bhowmik ${ }^{2}$, Sukriti Rana ${ }^{3}$, Payel Talukdar, ${ }^{4, *}$ Kalpana Datta $^{5}$ \\ ${ }^{1}$ Professor, Dept. of Medicine, Medical College Kolkata, ${ }^{2}$ Research Officer, Pediatric Centre of Excellence in HIV Care Medical \\ College and Hospital Kolkata, ${ }^{3}$ Resident, Lady Duffrin Victoria Hospital, Kolkata, ${ }^{4}$ Assistant Professor, Dept. of Psychiatry, Nil \\ Ratan Sircar Medical College and Hospital, Kolkata, ${ }^{5}$ Professor, Dept. of Pediatrics, Medical College and Hospital Kolkata, India
}

*Corresponding Author:

Email: drpayeltalukdar@gmail.com

\begin{abstract}
Community engagement in relation to HIV/AIDS has so often been a topic of discussion in India, substantial involvement of the community has rarely been a mainstream practice The general concept of community engagement is letting research projects achieve success by involving the participants directly into the research work, and implementing the results and the inferences obtained from those projects for the betterment of participants. We tried our level best to put this theory in practice at Medical College and Hospital, Kolkata, India during year 2015-2016. Apart from some teething problems that cropped up at the initial stage of the study, which was difficult and time taking to solve. The advantages of community involvement, as the experience showed, outweighed such minor difficulties. The final developed model for community engagement among marginalized population was successful and we can recommend this model for robust data collection and interpretation expecting a fruitful end result.
\end{abstract}

Keywords: HIV/AIDS, Community engagement, Community involvement.

\section{Introduction}

The menace of HIV/AIDS continues to threaten the very existence of human civilization worldwide especially the resource poor countries of the third world. The AIDS epidemic has infected more than 50 million people worldwide and its devastating effect is felt all the more painfully in the developing and the underdeveloped countries. The vulnerable population like women, children, young adults and the sexual minorities are the worst affected. ${ }^{1}$ The HIV epidemic in India is rapidly spreading from high risk urban group sex workers and IDUs to rural areas, particularly to housewives who had so far traditionally remained insulated from this infection and in this case their husbands being the migrant workers, happen to be the carrier of this deadly disease. India has more than 32 million people living with HIV positive and of whom women constitute about 45 percent. Poor economic status, low literacy level, lack of knowledge about HIV and very limited access to healthcare facilities are the major factors responsible for the proliferation of this disease among the female population in India.

Research on HIV/ AIDS is going on to combat the disease. Despite being a topic of much concern, HIV/ AIDS centric research in our country hardly went beyond the theme of prevention and management of the disease and on a very few occasions any attempt has been made to engage the community in these programs. It may also be noted that while community-engaged research programs are increasingly common in countries like the USA, Canada, and UK, only a few such programs have so far been conducted in India and that too mainly focused on prevention of HIV/ AIDS and other STIs, and evaluation of the issues related to the improvement of the quality of life and the quality of care taken for the people living with those diseases. ${ }^{2}$ When initiating a communityengaged research Programme researchers are challenged by the lack of any Single, standardized definition of a community. According to some authorities, the defining feature of a community is the common identity shared by its members. ${ }^{1}$ Thus a community can be defined in numerous ways including disease, ethnicity, geographic region, gender, etc. Community may be defined similarly, but experienced differently by people with diverse backgrounds. ${ }^{2}$ Given the complexity of the concept of community; it is not surprising that there is no universally accepted definition of community engagement. ${ }^{3}$ The Center for Disease Control (CDC) has defined it as the process of working collaboratively with and for groups of people affiliated by geographical proximity, special interest, or similar situations to address issues affecting the well-being of those people. ${ }^{4}$ In addition to defining the community, the general goals of community engagement in research may be diverse, e.g. to assess whether relevant research is culturally and practically acceptable, to ensure that community disruption is minimized, or, to avoid exploitation, by ensuring a fair distribution of the benefits of research. ${ }^{4}$

However, although community engagement in relation to HIV/AIDS has so often been a topic of discussion in India, substantial involvement of the community has rarely been a mainstream practice; and while community engagement would seem logical and humane, its acceptance has not been simple, owing to the current social, political and legal contexts and structures whereby the affected communities are marginalized ${ }^{5}$. Thus while the concept of community engagement has been recognized as an important component of the armamentarium in the war against HIV/AIDS; it has yet to be utilized effectively in India. In fact, community involvement has sometimes been described as only tokenistic, and often perfunctory. ${ }^{6-8}$ But recent empirical studies amply demonstrated the benefits of community engaged approach to research process and its outcomes that include:-

1. Improved participant recruitment

2. Enhanced capacity among research stakeholders

3. Productive conflict resolution

4. Increased quality of outputs and outcomes 
5. Increased sustainability of project goals beyond funding timeline etc. (AJPH policy) ${ }^{9}$

The community-engaged research programs, conducted so far, provided valuable inputs on the views, experiences and behavioral patterns of the community members in the course of their participation as a community. Voluntariness of an individual and the community, as espoused by a number of international guidelines, serves as a key to any bio-medical research. In the field of bioethics, it is generally presumed that a person acts voluntarily if he or she performs it intentionally without being under the controlling influence of another person or condition. ${ }^{9}$ It is really a Herculean task for every researcher to conduct HIV/ AIDS related research with full adherence to ethical norms which, among others, call for voluntariness of every participant and the feat is even more challenging in the multi-cultural setting of the country like India where family and social norms tend to mould the individual behavior.

Voluntariness of an individual and the community, as espoused by a number of international guidelines, serves as a key to any bio-medical research. In the field of bioethics, it is generally presumed that a person acts voluntarily if he or she performs it intentionally without being under the controlling influence of another person or condition. However, this concept of voluntariness is ill suited to explaining how certain social circumstances and the beliefs they conform might sometimes constrain their choices; owing to the fact that circumstances and beliefs might exert a controlling influence, causing them to feel pressured into performing certain actions, without necessarily removing their internal capacity for selfcontrol. ${ }^{10}$

In view of this backdrop it is therefore quite challenging to ensure full adherence to the standard ethical guidelines in developing and under-developed countries while conducting a research study. The guidelines has different aspects which include respect for person, beneficence, informed consent of the participants, maintenance of their privacy and confidentiality, riskbenefit analysis and participants' right to withdraw from the research or the trial at any point of time, without any penalty or loss of benefit, non-exploitation, participants' access to newly developed drug or device at post trial period etc. Last but not the least mandatory approval by ethics committees prior to the commencement of research project is an essential part of the standard ethical guidelines. $^{11}$

So, our endeavor to assess the voluntariness of consent to research of the women participants in a clinicbased HIV intervention trial in Kolkata, India was by no means an easy task given the complexity of the socioeconomic and cultural scenario in which the families grow and shape their value system.

\section{Diversity and Pluralism}

A huge challenge for community engaged research programs in India Being a country as large as a continent with its huge diversity in terms of language, ethnicity, religion, culture, food habit, political idiosyncrasies and so on India presents multiple challenges for communityengaged research programs. In the first place in a country like India with 22 official languages $14\left(8^{\text {th }}\right.$ schedule of constitution of India) and countless dialects, divided into as many as seven major religious groups (12) and each group having a number of sub-groups, no monolithic strategy of community engagement seems to succeed here. It is comparatively easy to work with the urban population who, though belonging to different cultural or social groups or castes, maintain and follow more or less uniform lifestyle. On the contrary, it is quite difficult to deal with the population living in rural areas with their allegiance and adherence to traditional culture and strict caste norms. Casteism or the caste system, which has been a custom of our society for centuries, is in fact a system of social stratification. This system, from time immemorial, separated communities into thousands of endogamous hereditary groups called 'jatis.

Secondly, a huge disparity in income level of men and women and moreover, the sheer dependence of women folk on the male members of the family regarding financial matters not only make them vulnerable in the society, but also reduces their views and opinions to insignificance. And this very trend, no doubt, was the biggest challenge for the researchers on their mission to ensure the voluntariness of participation.

Another major challenge we faced in course of our preliminary study was the all-pervading influence of political and religious leaders who tend to mould individual's behavior particularly rural and semi-urban areas. They intervene to settle family scores, and they can even keep any HIV positive patient or a rape victim completely isolated from the community. In utter defiance of and with little respect of the law of the country they very surprisingly and peculiarly enough run a parallel judicial system or the Kangaroo courts to keep the women folk subjugated.

Again the low literacy rate especially among the female population was also a stumbling block in our endeavor towards obtaining informed consent of the target people. In this context it may not be impertinent to mention that, According to 2011 census there was wide gender disparity in the literacy rate in India. Effective literacy rate (age 7 and above) in 2011 were 82.11 percent for men and 65.46 percent for women. Their poor literacy often comes in the way of ensuring informed consent as they could easily be misled into some wrong perception. Hence this population was chosen for the present study. ${ }^{12}$

\section{Constitution of Community Advisory Board}

A leap forward community-engagement. As the Community Advisory Boards (CAB) is meant to formalize the academic- community partnership we left no stone unturned to make our $\mathrm{CAB}$ all-inclusive with a view towards making different segments of the study population true partners in our research Programme a Community Advisory Board was formed involving the following personnel;

1. HIV/ AIDS affected persons (one male and two female)

2. The Secretary and the president of the state-wide network of NGOs that deal with HIV/ AIDS patients.

3. Independent researchers.

4. Psychiatrist 
5. Psychologist

6. Ethics Committee member

7. ART clinic staffs

8. NGO workers attending ART clinic

9. NGO leaders

This process was fraught with countless difficulties. And only with slow but determined steps did we overcome them.

\section{Challenges Encountered while forming the CAB}

At the time of formation of the Community Advisory Board we faced an initial resistance from the ethics committee members. Two of them simply refused to be the part of the Board citing the reason that, they had previously approved the research proposal when placed in the institutional ethics committee, how they themselves later can evaluate the ethical drawbacks of the same study. Later they were explained that, the research was regarding the evaluation of the voluntariness of the study participants and not specifically regarding the ethical approval of the research proposal. There continued to be a conflict of interest at last after a series of meetings held with Ethics Committee members we, at long last, succeeded in getting the involvement of one of them.

If this job was quite a tough one, the next one was too by no means less difficult - that was to take the NGO leaders into confidence and address their skepticism about our motives, including their apprehensive of our 'evil' design supported by raising foreign grants to manipulate the patients as guinea pigs. It was a painstakingly long process involving a series of discussions and meetings to convince them that the project was not about any drug trial, nor did it promise any magic pill to cure the disease overnight. But that in fact it was to study the ways in which we might give Indian women living with HIV/AIDS more power in deciding whether they wished to participate in research studies. We also made it clear that the research would not result in any short-term gain for patients but rather, would, in the long- run ultimately benefit the target group - the population living with HIV/ AIDS. We were, to some extent, successful in our endeavor when some of the skeptical NGO leaders became extremely enthusiastic in due course and confessed that they had never seen any research project of the like. They went further to being deprived of the facilities of the organized sector, medical insurance etc. these poor patients, on their part, solely depend on these handful NGOs for their welfare needs including free treatment, employment etc. Their total dependence on the NGOs, on the other hand, renders them vulnerable to the dictates of the NGO leaders.

Another sticky issue we had to face when some of the $\mathrm{CAB}$ members, especially the researchers, argued in favor of including religious leaders or local headmen in the Board as they were, the researchers said, expected to play the role of ' Gate keepers' or 'Watchers' in any research project. The Patients' representatives, on the other hand, vehemently opposed the proposal.

As those leaders, the representatives argued, being unfortunately ignorant of various avenues through which the infection could spread in human body other than actual sexual activities, such as blood transfusion and as the disease was still looked down upon as symbol of 'depravity' in our male-dominated, repressive social system that was governed by some overtly puritan minds, the inclusion of religious leaders might pose a threat to the female participants and we readily accepted this argument. As our target group consisted of women belonging to different religious groups it was decidedly a wise approach, on our part, not to include any religious leader in the Community Advisory Board.

The other members of the $\mathrm{CAB}$ readily agreed to the proposal and helped to strengthen the $\mathrm{CAB}$ and was involved in the study, being aware of their roles and responsibilities as explained to them during their recruitment procedure.

\section{Next steps taken}

After the differences were sorted out nitty-gritty of our study was placed before the members of the $\mathrm{CAB}$ and their opinions were sought. They were involved in overall planning, questionnaire framing, as well in the recruitment process of researchers and interviewers.

After the pilot study we took up the questionnaire which was rendered from English into Bengali, the native language of the participants, by a bi-lingual expert for the revision of content and language. In keeping with the suggestions of our Board members, especially those who represented the patients themselves, we transformed the questionnaire into a more self-explanatory one by infusing terms and jargons used by the common people in their day-to-day business for example, assuming their low rate of literacy and poor comprehension level we chose to use the ana scale. Ana, the denomination of currency formerly used in local market has made its way in local dialect too, where 4 ana stands for 25 percent and 8 ana for 50 percent and so on. We also back translated the questionnaire into English and finally got it examined three other bi-lingual experts to make sure that our process was foolproof.

As a part of our endeavor to validate the efficacy of the questionnaire for making an impact on the participants we presented another pilot study for CAB approval.

The Results of the pilot study served as an eye opener for us. Despite the questions being read out to most of the participants who expressed their inability to read and write, they failed to mark their preferences on the Ana scale in a meaningful and logical manner, every time choosing a different option for the same question which pulled down the credibility level almost to naught. Our previous experience had made it clear that our participants did not possess great power of arithmetical comprehension. Hence, they even failed to differentiate between two denominations as simple as 3 and 4 or 5 and 6. Given the low literacy of our target population this failure was not something quite unexpected for us. So, once again we took the matter to the CAB members. This time they suggested including a set of illustrations in the questionnaire to create a greater appeal to the perception level of the participants. This suggestion was very successful as the participants there after accomplished the assigned task of marking their preferred numerical choice with a more clear precision taking the reliability of the second pilot study to the near perfect level. 
Patient recruitment Process - the next step towards community-engagement.

Encouraged by the success of both the pilot studies we moved forward to the most crucial phase of our Programme-recruitment of patients for in-depth interview and focus group discussion. In this process we strictly adhered to traditional path of community engagement and involvement and sought the help of NGO headmen we had appointed in our CAB and engaged with them in our overall planning process.

NGO leaders exert a considerable influence on our target people, the population living with HIV/ AIDS and a sizable portion of which is receiving patronage from them, through their state-wide network and play a significant role in moulding and twisting the views and opinions of these people. So, we thought it wise to abide by their suggestion as far as the recruitment process was concerned.

Contrary to their assurance, they insisted on the inclusion of the patients of their choice whom they groomed as good 'orators' and thus tend to reduce our scope for random sampling. It was a painstakingly long process, on our part, to make them convinced of our need for random sampling, as we required the patients in their real surroundings, and not the good ' orators', who were capable of juggling the truthful information.

The inclusion of the NGO leaders of the CAB in the patient recruitment process not only made our task easy, but also dispelled the cloud of doubt from the collective psyche of the prospective participants and inculcated a sense of belonging in them.

We identified 60 participants of parent study who may be approached for our study. We approached them with the help of CAB members. Date, time and venue of the interviews were also finalized after a brief meeting with $\mathrm{CAB}$ members and a place was hired for the same at a neutral location. CAB members, particularly the NGO leaders and the patients' representatives placed special emphasis on employing female interviewers belonging to nearly same age group as that of participants and capable of interacting with the participants in their local dialect without being too prudish.

Accepting the suggestions of the $\mathrm{CAB}$ members we engaged a good number of female researchers who were entrusted with the sole task of interviewing the patients and finally conducting the FGDs. The male doctors who were in charge of overall supervision and execution of the project, stepped aside as much as possible from the process of day-to-day interaction with the patients and participants.

Based on $\mathrm{CAB}$ recommendations the Focus Group Discussions (FGD) were also conducted by the female researchers. A total of four FGDs were conducted- two with HIV seropositive female volunteers to evaluate their degree of voluntariness to participate in an HIV medical adherence behavioral intervention and two with HIV seropositive males to get a clear picture of the power structure that exists in our society as it relates to informed consent.

In all these interactive sessions the $\mathrm{CAB}$ members consisting of HIV/ AIDS affected persons, the Secretary and the president of the state-wide network of NGOs that deal with HIV/ AIDS patients, independent researchers, psychiatrist, psychologist, ethics Committee member , ART clinic staffs and the NGO workers attending ART clinic stressed the importance of using local dialect for easy communication and the presence of the translator who was entrusted with task of translating the transcript of the FGD from Bengali to English during each interactive session. The presence of translator paved the way for an easy translation of the FGD transcript while keeping the essence of the original language and the colloquial flavor and vigor intact.

The presence of the translator helped to prevent the interruption of semantic noise from the open communication process. A word or a phrase may not necessarily carry the same unmistakable connotation to all and tend to disrupt the communication process.

\section{Conclusion}

From the very inception of our project we were exposed to countless hurdles and faced resistance from all quarters. Without the help and co-operation extended by the $\mathrm{CAB}$ members we could not have reached our goals. But the things were not as smooth as it appeared at the initial stage. Braving so many odds we have come a long way only with the support extended by our community. Our arduous effort of community engagement paid off when we saw a lot of women infected with HIV positive were coming forward to participate in our Programme. They were not even ashamed to share their views and experiences with the researchers which in turn facilitated the researchers' task. They were gradually overcoming the apprehension of social stigma which had so far rendered them socially vulnerable. As our research is still in progress, it is too early to draw any conclusion or predict its final outcome. But after a long tireless journey a pleasant sense of achievement engulfed us when a young girl from a village who took the trouble of covering almost $200 \mathrm{KM}$ to have her ART in this center firmly proclaimed: 'I will no longer feel ashamed and next time I will seek my treatment from a nearby hospital. I am well aware that there are people who will despise me, ouracize me, but I will remain undaunted...' We feel that we are a step forward to our goal and are definitely on the right track.

Thus with this long endeavor we think we could develop a model for community engagement among marginalized population successfully and we can recommend this model for robust data collection and interpretation expecting a fruitful end result.

\section{References}

1. Peter R. Lamptey, Jami L. Johnson, and Marya Khan, "The Global Challenge of HIV and AIDS," Population Bulletin 61 , no. 1

2. WHO. Geneva. A primer for mainstreaming health promotion. Working draft for The Nairobi Global Conference on Health Promotion, Kenya. 2009. Oct, [Last accessed on 2017 Apr 7].

3. Vallely A, Shagi C, Kasindi S, Desmond N, Lees S, Chiduo B, et al. for the Microbicides Development Programme. The benefits of participatory methodologies to develop effective community dialogue in the context of a microbicide trial feasibility study in Mwanza, Tanzania. BMC Public Health. 2007;7:133. 
4. Centers for Disease Control and Prevention Principles of community engagement (1st ed)Atlanta (GA): CDC/ATSDR Committee on Community Engagement; 1997

5. Muthuswamy V. Ethical issues in HIV/AIDS research. Indian J Med Res, April 2005:121;601-610.

6. Chipuer, H. M., \& Pretty, G. M. H. Journal of Community Psychology, 1999;18:83-115. A review of the Sense of Community Index: Current uses, factor structure, reliability, and further development. Long, D.A., \& Perkins, D.D. Journal of Community Psychology, 2003;27(6), 643-658.

7. Confirmatory Factor Analysis of the Sense of Community Index and Development of a Brief SCI. Journal of Community Psychology, 31, 279-296

8. Community Engagement Step-by-Step Action Kit, Gottlieb, Hildy, 19-20 (2007)

9. Donna Jo McCloskey, RN, PhD, (Chair), Mary Anne McDonald, DrPH, MA, Jennifer Cook, MPH, Suzanne
Heurtin-Roberts, PhD, MSW, Stephen Updegrove, MD MPH, Dana Sampson, MS, MBA, Sheila Gutter, PhD, Milton (Mickey) Eder, PhD: 'Definitions and organizing concepts from literature'

10. Sergio Aguilar-Gaxiola, MD, PhD, University of California, Davis (Co-Chair) J. Lloyd Michener, MD, Duke University (Co-Chair): Principals of Community Engagement (Second Edition)

11. http://ajph.aphapublications.org/userimages/ContentEditor/1 432646399120/authorinstructions.pdf

12. http://censusindia.gov.in/2011common/censusdataonline.ht $\mathrm{ml}$ 